\title{
STAINING PLASTIC BLOCKS WITH TRIIODIDE TO IMAGE CELLS AND SOFT TISSUES IN BACKSCATTERED ELECTRON SEM OF SKELETAL AND DENTAL TISSUES
}

\author{
A. Boyde \\ Dental Physical Sciences, Oral Growth and Development, Dental Institute, Barts' and The London School of \\ Medicine and Dentistry, Queen Mary University of London, London E1 4NS, UK
}

\begin{abstract}
Backscattered electron scanning electron microscopy (BSE SEM) is an invaluable method for studying the histology of the hard, mineralised components of poly-methyl methacrylate (PMMA) or other resin embedded skeletal and dental tissues. Intact tissues are studied in micro-milled or polished block faces with an electron-optical section thickness of the order of a half to one micron and with the area of the section as big as a whole - large or small - bone organ. However, BSE SEM does not give information concerning the distribution of uncalcified, 'soft', cellular and extracellular matrix components. This can be obtained by confocal microscopy of the same block and the two sorts of images merged but the blocks have to be studied in two microscope systems. The present work shows a new, simple and economic approach to visualising both components by using the triiodide ion in Lugol's iodine solution to stain the block surface prior to the application of any conductive coating - and the latter can be omitted if charging is suppressed by use of poor vacuum conditions in the SEM sample chamber. The method permits the use of archival tissue, and it will be valuable in studies of both normal growth and development and pathological changes in bones and joints, including osteoporosis and osteoarthritis, and tissue adaptation to implants.
\end{abstract}

Keywords: Bone; cartilage; osteoid; histopathology; implant interface.

*Address for correspondence:

Alan Boyde

Dental Physical Sciences, Oral Growth and Development, Dental Institute

Barts' and The London School of Medicine and Dentistry Queen Mary University of London, London E1 4NS, UK Telephone Number: +44 (0) 2078825984 E-mail: a.boyde@qmul.ac.uk

\section{Introduction}

Polished or micromilled surfaces of poly-methylmethacrylate (PMMA) or other plastic embedded bone blocks have been extensively employed for scanning electron microscopy (SEM) using backscattered electrons (BSE) to visualise the mineralised regions and quantify their mineral content. BSE SEM gives a compositional signal level dependent on mean backscattering coefficient of the embedded tissue, which is evidently much higher in the mineralised skeletal and dental tissues. Measuring the electron backscattering coefficient by reference to standard materials provides a means of comparing mineral concentration (Boyde et al., 1992a; Boyde et al. 1999b; Boyde et al., 2005). The information depth ( $\mathrm{Z}$ resolution) under typical circumstances of $20 \mathrm{kV}$ electron bombardment is about one half micron, and, as such, gives a much thinner electron optical section in the block surface than could be produced by any method for physical sectioning over large fields. Sideways scattering limits the XY resolution too, but this is easily better than can be achieved by light microscopy (LM) or confocal scanning light microscopy (CSLM). However, BSE SEM of such tissue usually provides no useful contrast due to cells, including sub-cellular features such as nuclei, or the unmineralised extracellular organic matrix of the hard tissues (for example, osteoid, predentine, hyaline cartilage), or bone marrow, or blood vessels, or the surrounding soft tissues such as periosteum, tendon, ligament, joint capsule and muscle. These can all be stained with organic dyes applied to the block surface and the surface layer imaged with transmitted light microscopy (LM) or by epifluorescent illumination to make the blockbulk self-luminous, thus back-lighting the stained layer in the block surface (Bab et al., 1984). Alternatively, the soft tissue histology can be studied by autofluorescence mode CSLM, when the block surface layer can be selected to make the information depth limited to something like the layer which we image with BSE SEM. Then the two types of digital imaging can be joined to allow the CSLM to fill in the blank, black spaces in the SEM images (Boyde et al., 2005).

It would obviously be more convenient to image both soft (unmineralised) and hard (mineralised) regions in the one microscope system. This is done in conventional LM sectioned-tissue histology by using staining procedures for the mineral component (e.g., von Kossa) or by differential staining for previously mineralised or unmineralised regions in decalcified tissue. In transmission electron microscopy (TEM), heavy metal staining is in almost 
ubiquitous use, and indeed any tissue stained en bloc for TEM can be studied by BSE SEM of the block face. Equally, tissue prepared for otherwise routine SEM study can be stained with a heavy metal (Newcomb and Boyde, 1980). Stains are often applied to ultra-thin sections for TEM after they have been cut, so the resins employed are clearly permeable to ionic diffusion over the required distances of about one twentieth of one micron.

The problem area that we address here is the utilisation of extant, archival material prepared without any heavy element staining. In the present study, it was decided to test triiodide (Lugol's iodine) as a block surface stain for SEM and it has been such a considerable success that we feel that others might profit by this knowledge. Other synonyms and abbreviations for this stain are IKI, iodinepotassium iodide, Lugol's solution, microiodide, potassium triiodide, potassium iodide (K(I3)), Bouchardat's reagent, and Gram's iodine.

\section{Materials and Methods}

Samples comprising bone and surrounding tissues were fixed in $70 \%$ ethanol, or in $10 \%$ neutral formol saline (NFS) for $24 \mathrm{~h}$ and then stored in $70 \%$ ethanol, or in some instances stored deep frozen and then fixed in $70 \%$ ethanol on thawing. Whole bones or slices were dehydrated in ethanol and embedded in polymethylmethacrylate (PMMA) and the block surfaces trimmed and polished or diamond micro-milled. Blocks which had been previously carbon coated to prevent charging in a conventional SEM were repolished to remove the coating.

\section{Staining procedure}

A $7.5 \%$ stock solution of iodine in potassium iodide was applied neat by pipetting directly on to finished PMMA block surfaces, leaving the solution in place for from $2 \mathrm{~min}$ to $15 \mathrm{~min}$ (Fig. 1). Alternatively, it was diluted by adding about $50 \%$ as much ethanol prior to use (Figs. 2-13). At the end of this period the stain was washed off with distilled water and the block blotted dry with absorbent paper.

\section{SEM}

Stained blocks were imaged using $20 \mathrm{kV}$ BSE in a Zeiss EVO MA10 SEM, uncoated, using a chamber vacuum of $49 \mathrm{~Pa}$. Figure 1 was obtained using a probe current of $1.4 \mathrm{nA}$ and Figs. 2-13 using $1.0 \mathrm{nA}$. Working distance (WD) was in the range 8 to $12 \mathrm{~mm}$.

\section{Examples of material studied with the triiodide method.}

1. Small bones including growth cartilage and soft tissue

- Mouse tails (Fig. 1).

- Rat distal femur (Figs. 2-4).

2. Large bones with substantial masses of hyaline cartilage - 2 year old Thoroughbred (TB) racehorse third metacarpal bones from treadmill and racetrack exercise training experiments (Boyde et al., 1992a; Boyde et al., 1997; Boyde et al., 1999a: Figs. 5-9).

- Icelandic horse tarsal joints (from Charles Ley, SLU Uppsala: Fig. 9).

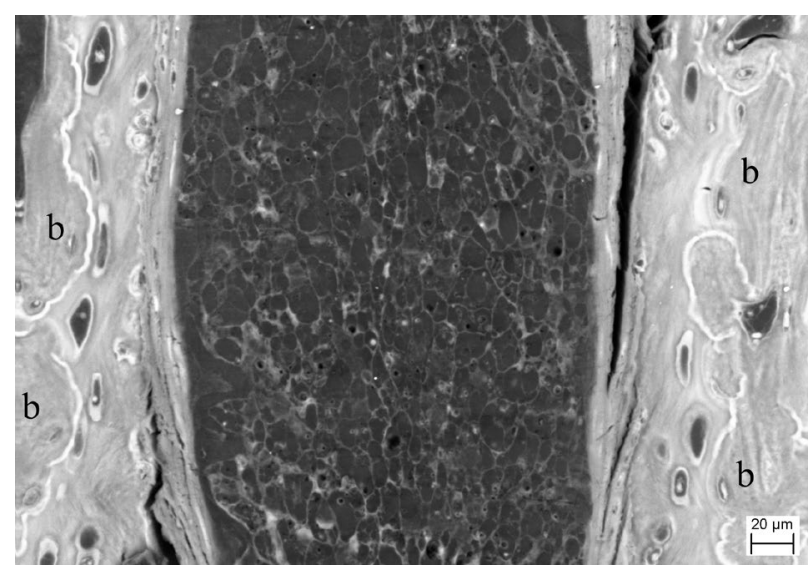

Fig. 1. Mouse tail intervertebral joint, showing nucleus pulposus and adjoining end plate cartilage and bone (b). Aqueous triiodide stained. WD $=11.5 \mathrm{~mm}$. Width $=390 \mu \mathrm{m}$. Probe current $=1.4 \mathrm{nA}$.

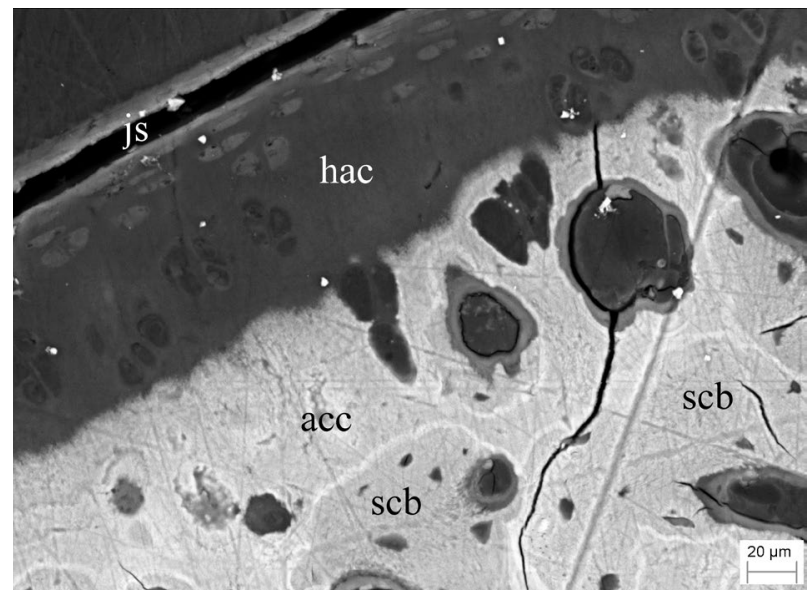

Fig. 2. Rat distal femur condylar cartilage: js = joint space cleavage in PMMA: hac = hyaline articular cartilage: acc $=$ articular calcified cartilage: $\mathrm{scb}=$ subchondral bone. Ethanol diluted Lugol's iodine stained, as are all samples shown in following figures. $\mathrm{WD}=9.0 \mathrm{~mm}$. Width $=342 \mu \mathrm{m}$. Probe current $=1.0 \mathrm{nA}$, same for remaining figures.

- Elephant 'sixth digit' (Hutchinson et al., 2011: Fig. 10).

3. Implant sites and surrounding tissue

- Human jaw dental implant site trephine cores (Wong, 2000: Fig. 11).

- Implants in rabbit tibia (Bonfield et al., 1981; Boyde and Wolfe, 2000: not illustrated).

- Dog maxillae (Figs. 12 and 13) with dental implants (not illustrated).

\section{Results}

The reason for adding ethanol in initial experimentation was to improve wetting and permeability of the PMMA surface. However, useful staining with triiodide was observed in every case with more intense staining from the concentrated aqueous solution (Fig. 1) than from the diluted 

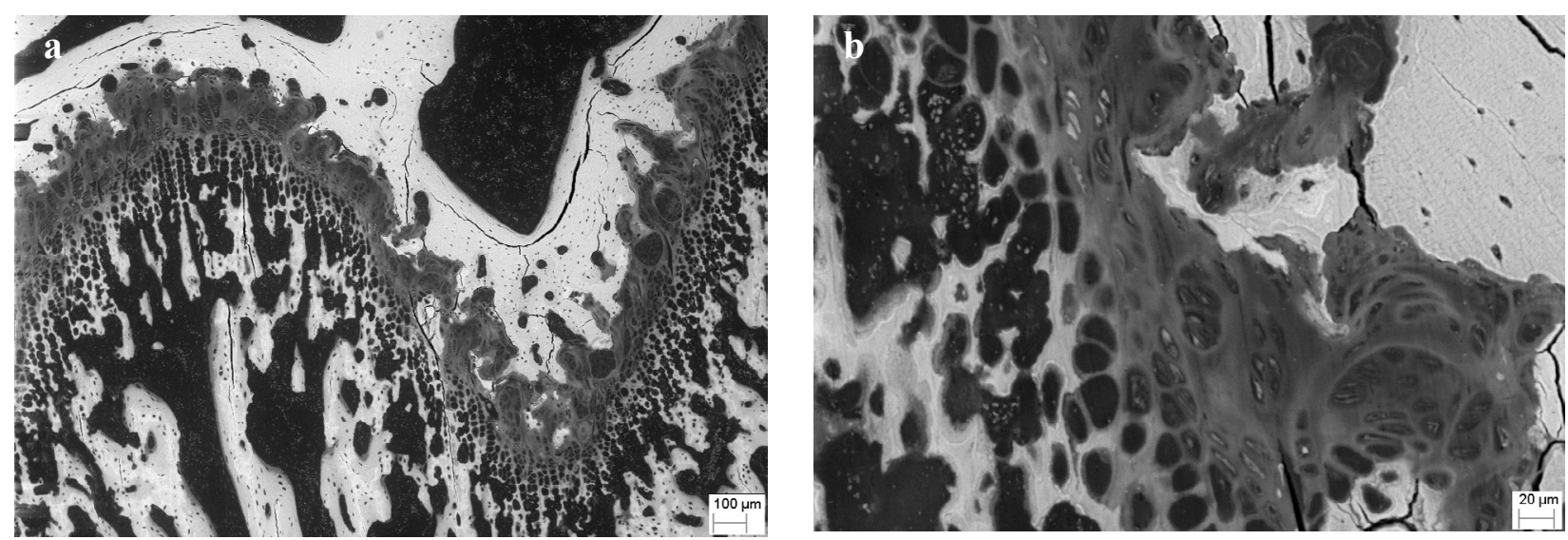

Fig. 3. Rat distal femur growth plate. (a) WD $=10.0 \mathrm{~mm}$. Width $=2.256 \mathrm{~mm}$, (b) WD $=10.0 \mathrm{~mm}$. Width $=428 \mu \mathrm{m}$.

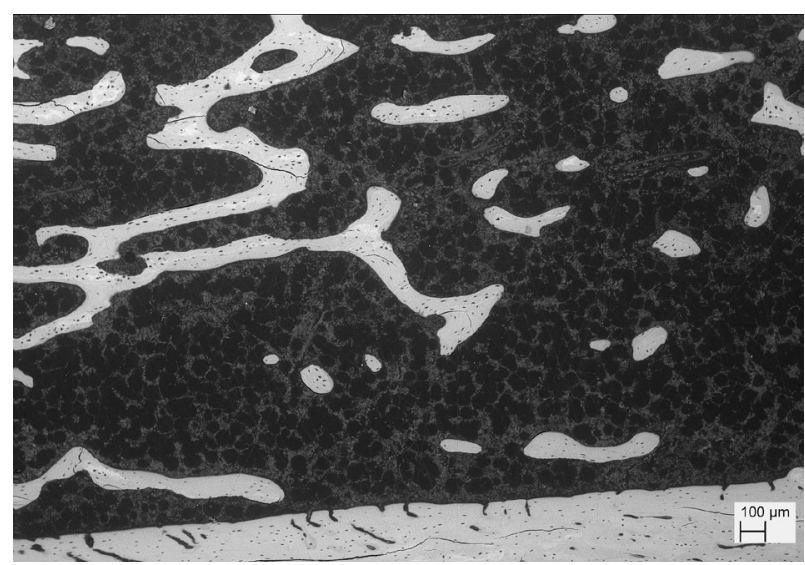

Fig. 4. Rat distal femur metaphyseal region showing mixed haematopoietic and fatty marrow. Large black spaces are adipocytes. Cortex below. WD $=9.0 \mathrm{~mm}$. Width $=3.171 \mathrm{~mm}$.

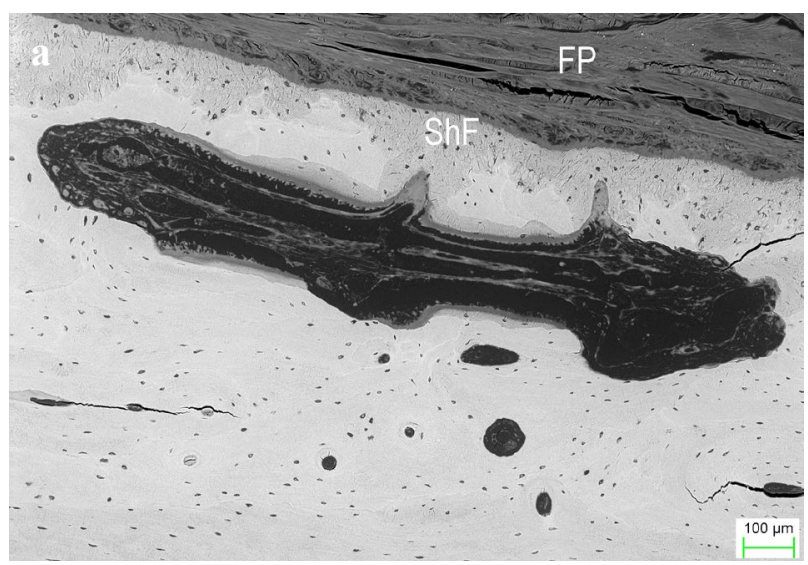

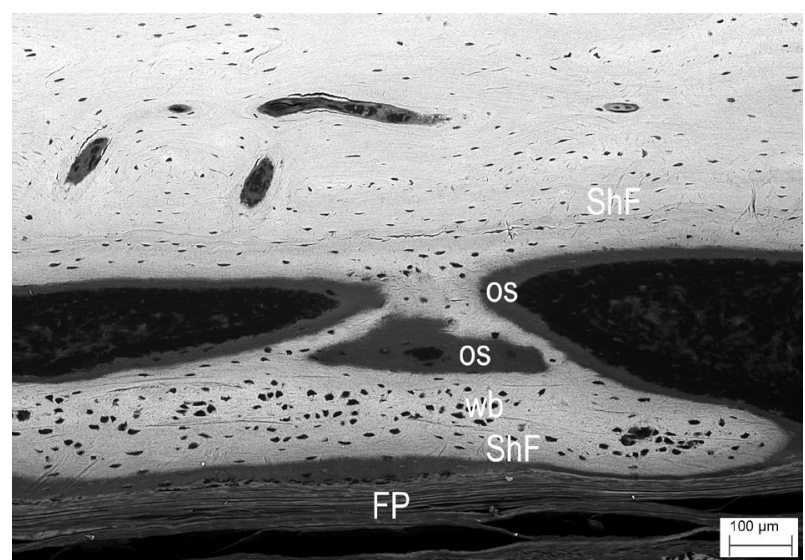

Fig. 5. TB racehorse distal third metacarpal bone, cut longitudinally, showing plexiform new bone formation on cranial cortex. $\mathrm{FP}=$ fibrous periosteum. $\mathrm{wb}=$ woven bone. $\mathrm{os}=$ osteoid. $\mathrm{ShF}=$ Sharpey fibres. $\mathrm{WD}=10.0 \mathrm{~mm}$. Width $=1.262 \mathrm{~mm}$.

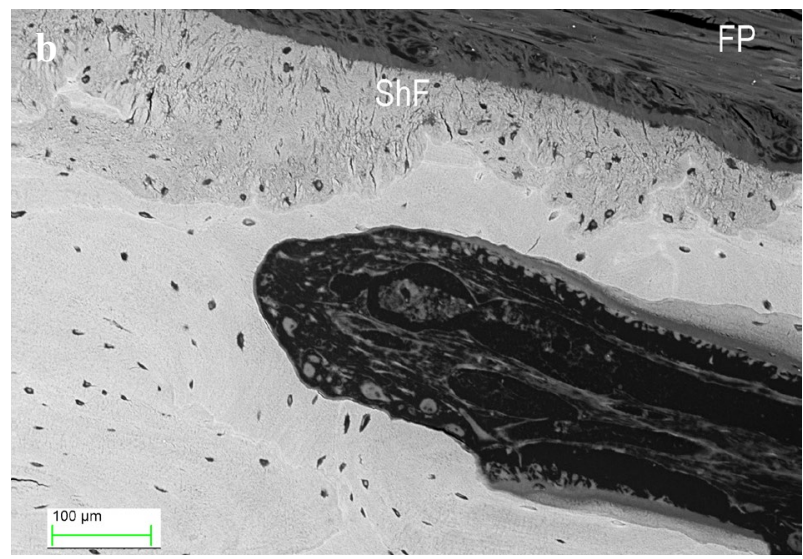

Fig. 6. (a) TB racehorse distal third metacarpal bone, caudal cortex, cut longitudinally, showing double ended cutting cone, obviously advancing to left (note several osteoclasts) and having recently advanced to right. FP $=$ fibrous periosteum. $\mathrm{ShF}=$ Sharpey fibres. $\mathrm{WD}=9.0 \mathrm{~mm}$. Width $=1.564 \mathrm{~mm}$; $(\mathbf{b})$ Higher power view of advancing edge of cutting cone seen in left of Fig. $6 \mathrm{a} . \mathrm{WD}=9.0 \mathrm{~mm}$. Width $=804 \mu \mathrm{m}$.

ethanolic solution (Figs. 2-13). When the concentrated aqueous stain was used, crystalline deposits were sometimes observed on the surface of the block outside tissue components within the PMMA, and excessively heavy staining and/or accumulation of precipitated stain occurred in defects in the block surface, such as 'blows' in the methacrylate and in the fine cracks which develop upon prolonged storage at the surface of polished blocks.

The bulk soft tissue components in PMMA 'bone' blocks stained with triiodide included muscle, dense fibrous connective tissue (collagen in fibrous periosteum, tendon, ligament, annulus fibrosus), sparsely cellular 


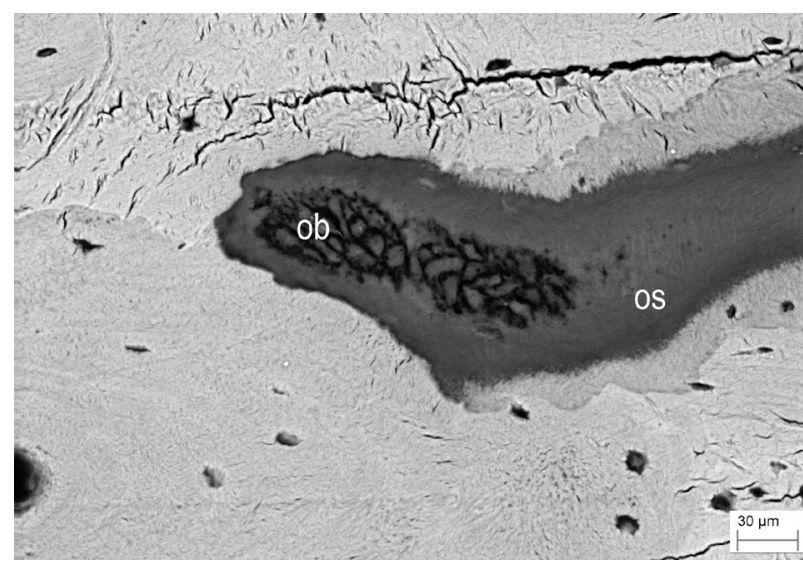

Fig. 7. TB racehorse distal third metacarpal bone, caudal cortex, cut longitudinally, showing closing region of secondary osteone (cutting cone) in tangential section close to its wall. $\mathrm{ob}=$ osteoblasts. os $=$ osteoid. Orientation of collagen fibrils in osteoid can just be discriminated. WD $=9.5 \mathrm{~mm}$. Width $=394 \mu \mathrm{m}$.

regions (nucleus pulposus, bone marrow) and articular and growth plate cartilage. Elastic laminae in small arteries and arterioles stained very intensely (not illustrated). Striated muscle stained more than bulk collagen, permitting their distinction in low magnification images.

At the cellular resolution level, nuclei generally stained more intensely than cytoplasm but could not always be distinguished. Osteoblasts and osteoclasts could be easily recognised where the tissue preservation was good enough. Cellular preservation was, as would be expected, generally very poor in large bone masses which had been stored deep frozen and had not been formaldehyde or ethanol fixed at the time of tissue acquisition. Nevertheless, the ability to see and read cartilage matrix histology proves to be very useful in its own right.

Details of histology surrounding metal implants in dog jaw bone and hydroxyapatite reinforced polyethylene implants (Bonfield et al., 1981) in rabbit tibia (not illustrated) and within trephine cores taken from human jaws prior to implant placement show that BSE imaging of triiodide staining will also provide a useful new tool in the armamentarium for studying implant-tissue interfaces.

The triiodide stain can be applied to block surfaces which had been previously stained with LM stains such as toluidine blue (Bab et al., 1984). The triiodide stained blocks could also be imaged with CSLM fluorescence after SEM study. The exact fields which had been studied in the SEM can be identified (at least in narrower fields corresponding to higher SEM magnification) by the change in fluorescence intensity at the 'left hand' edge of the SEM field: this line is caused by the extra dwell time of the electron beam and therefore excess radiation damage to the sample at the beginning of each line in the SEM image.

\section{Discussion}

We have previously used iodine as a negative electron stain by embedding tissue in an iodinated methacrylate (Boyde

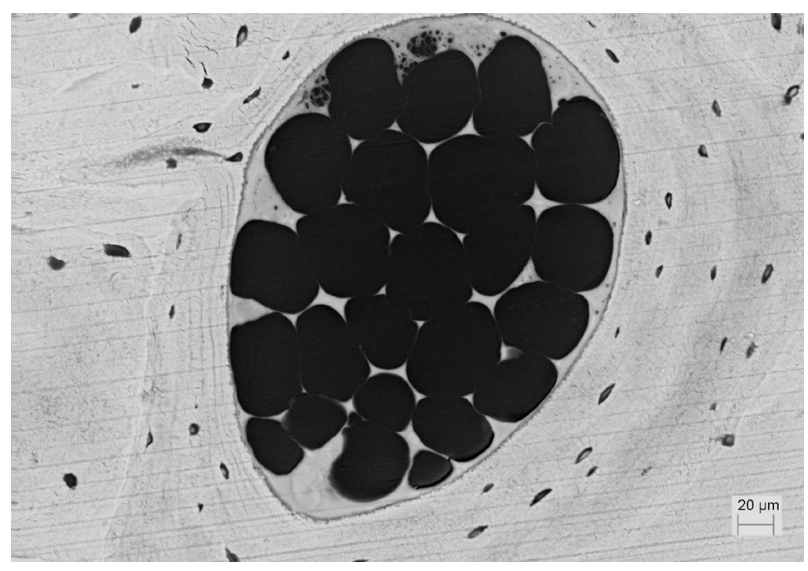

Fig. 8. TB racehorse distal third metacarpal dense condylar bone, showing canal space almost entirely filled with adipocytes which appear black because fat content was replaced with PMMA during embedding. Other small back features in clusters are erythrocytes in capillary blood vessels. $\mathrm{WD}=9.0 \mathrm{~mm}$. Width $=446 \mu \mathrm{m}$.

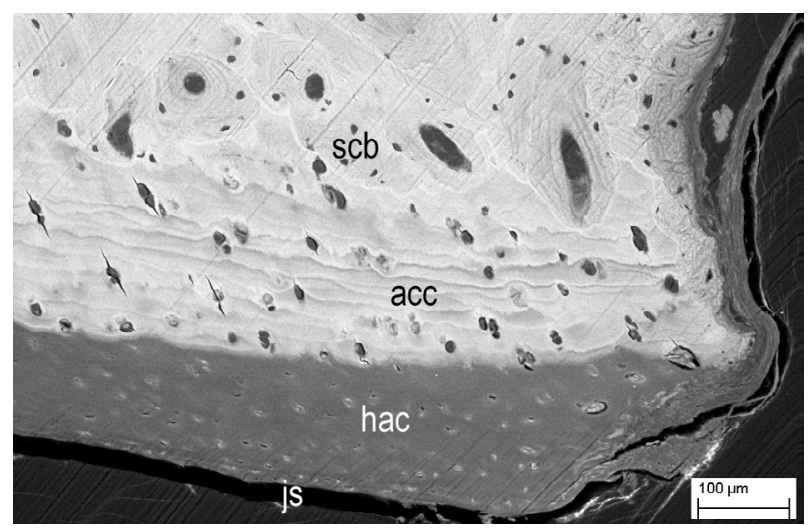

Fig. 9. Icelandic horse tarsal bone: js = joint space cleavage in PMMA: hac $=$ hyaline articular cartilage: acc $=$ articular calcified cartilage: $\mathrm{scb}=$ subchondral bone. WD $=9.0 \mathrm{~mm}$. Width $=869 \mu \mathrm{m}$.

et al., 2001b; Boyde and Koole, 2001). We have also used halogenated methacrylates as standards in quantitative BSE SEM studies (qBSE; Boyde et al., 1999b; Boyde and Firth, 2005).

Several authors have recently reported the use of triiodide as a bulk stain for x-ray microtomography $(\mu \mathrm{CT}$ : Metscher, 2009a; Metscher, 2009b; Degenhardt et al., 2010; Ritman, 2011). Stephenson et al, (2012) showed differential staining of the cardiac conducting system. Cox and Jeffery (2011) and Jeffery et al. (2011) showed useful differential staining of striated muscle and dense connective tissue and more intense staining of muscle was also found with the block surface staining reported here. As with these microtomographic studies, triiodide staining can no doubt be conducted prior to embedding, but the advantage of the present simple method is that we can use existing experimental material which has been permanently preserved by PMMA embedding, and that we can conduct quantitative BSE for mineral content determination with the same blocks, prior to staining . 

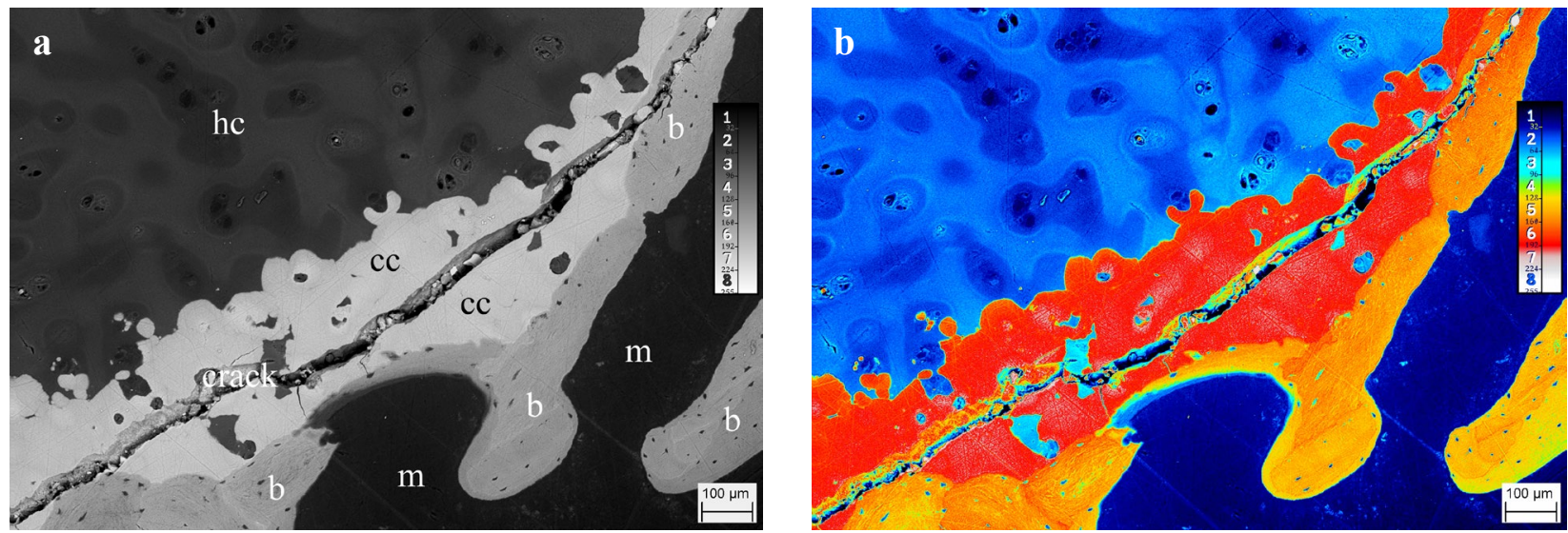

Fig. 10. (a) Indian elephant predigit BSE SEM of PMMA embedded slab at calcified cartilage:bone interface where densest phase is calcified cartilage (See Hutchinson et al., 2011). Triiodide stain shows histology in unmineralised hyaline cartilage; (b) Because of the great contrast range it is easier to recognise tissues in a pseudo-coloured version (look up table shown in the inset). Labelling in Fig. 10a: hc = hyaline cartilage: $c c=$ calcified cartilage: $b=$ bone: $\mathrm{m}=$ marrow space in bone. $\mathrm{WD}=11.0 \mathrm{~mm}$. Width $=1.504 \mathrm{~mm}$.

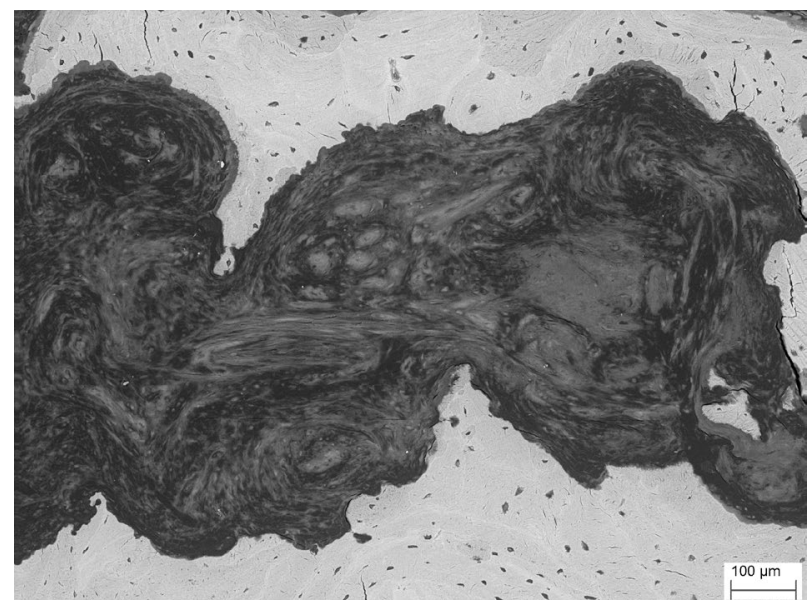

Fig. 11. Bone sample retrieved from trephine drill core for implant placement from human mandible, showing detail of histology in bone marrow. WD $=9.0 \mathrm{~mm}$. Width $=1.222 \mathrm{~mm}$.

The set of methods which employ PMMA blocks can now be extended. We can use the blocks for CSLM for 3D histology deep to the block surface and for imaging tetracycline, calcein and alizarin mineralising front labels. Next, we can study mineral concentration by qBSE imaging (e.g. Boyde et al., 1992a; Boyde et al., 1999b; Boyde and Firth, 2005). Images from these two methods can be correlated (Boyde et al., 2005). Previously, we would then have used the blocks to prepare casts of the nonmineralised space compartments, both marrow space and blood vessel canal spaces in wider field and the osteocyte lacunar-canalicular system in higher magnification images (Boyde, 2012). Now we can add the intermediate step of reading the cellular and matrix histology in the very same layer from which we obtain the BSE image of the mineral component and in one and the same image.

One of the most useful features of triiodide staining to permit the reading of non-mineralised histology in BSE imaging in the SEM in our field of endeavour is the

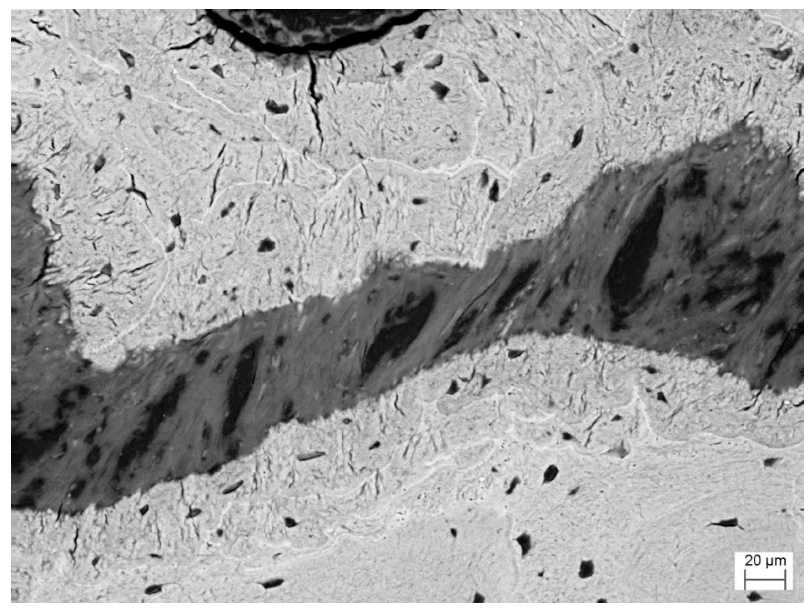

Fig. 12. Dog maxillary-premaxillary suture showing Sharpey fibres connecting the two bones. WD = $11.5 \mathrm{~mm}$. Width $=396 \mu \mathrm{m}$.

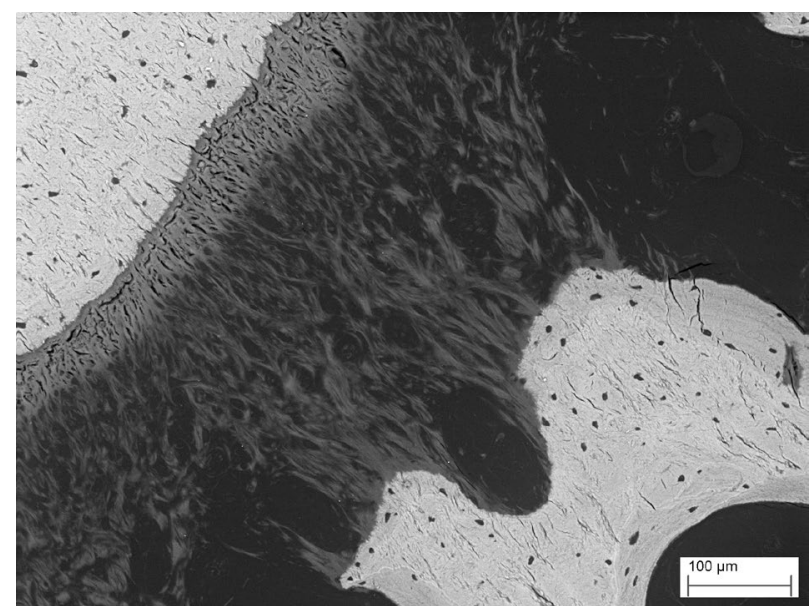

Fig. 13. Dog maxillary canine periodontal ligament showing Sharpey fibres connecting bone (lower right) to tooth cementum (upper left). WD $=9.0 \mathrm{~mm}$. Width $=772 \mu \mathrm{m}$. 
ability to study joint cartilage. This will open up the study of animal models of osteoarthritis (OA) as well as human OA. We may now easily distinguish hyaline cartilage from pure PMMA in the BSE image. One can just achieve this also with unstained blocks, but the lower grey level for soft tissue is then so far from the upper level of calcified cartilage - normally the most densely mineralised tissue in a bone organ - that the range of grey levels within the calcified tissues is not properly represented. The best prior known method for studying cartilage distribution as such using BSE SEM is to use the A-B topographic contrast mode (Ferguson et al., 2003).

The aim of this study was to develop a method for combining SEM imaging of hard tissue with adequate visualisation of associated soft tissues. This is of obvious importance in its potential to empower significantly the anatomical and pathological study of skeletal tissues. BSE imaging is the gold standard for measuring mineral content in bone, which is of obvious relevance in a number of bone diseases, but, until now, osteoid seams had to be imaged and measured separately by light microscopy. Sequential use of qBSE imaging of unstained PMMA blocks followed by resurfacing and triiodide staining could bring together all the qualitative and quantitative assessments one needs to make on bone pathological samples in one single method, imaging system, and approach.

Penetrability of the plastic block to the triiodide stain will vary widely with the quality of the embedding - the poorer the embedding, the more rapid the penetration and staining. Glaring over-staining only occurred with the concentrated aqueous stain and might be avoided by immersion in a diluted solution or by judicious rinsing after staining. Ethanol and ethanolic solutions wet PMMA far better than does water, so that it is easier to apply a discrete drop of stain from an aqueous solution. This will sometimes be desired when it is wished not to alter the backscattering properties of the entire block surface.

The quality of embedding will be suspect if the tissue appears opaque rather than translucent, which will equate with a strong reflected light signal seen with CSLM or a steep reduction in the strength of any fluorescence signal in the CSLM with depth focussed into the tissue. Under these circumstances, it would be sensible to wet the block with say $70 \%$ ethanol for a few minutes prior to the application of the stain solution in order to prevent stain immediately penetrating into microporosities in the block during the wetting phase, such stain then precipitating on the surface and in surface defects during the drying phase.

It would be very convenient if an iodine containing stain could be employed which could be visualised in either LM or CSLM and in the SEM. As such, erythrosine was tested in the present study. However, although heavily stained tissue regions such as hyaline articular cartilage could be seen with BSE SEM, the intensity of the fluorescence image deep to the block surface was largely extinguished by absorption of light in the extremely densely stained surface. More work needs to be done in this area.

Another approach to seeing a fluorescent stain in both SEM and LM is to use the cathodoluminescence (CL) signal in the SEM, and this works well enough for samples which are stained prior to embedding with a rather electron beam stable CL stain like brilliant sulphaflavine (Boyde et al., 1983). The snag here is the need to have a good CL detector which will work with a good BSE detector, and that one cannot just use archival, unstained PMMA material. Tetracycline lines in calcified tissues are, however, nicely shown by CL SEM (Boyde and Reid, 1983; Boyde et al., 1992b).

\section{Conclusions}

Triiodide block surface staining for SEM is a useful new method which will lead to contributions to basic knowledge and understanding in bone and joint biology and pathology. Of particular importance is the ability to study osteoid, bone marrow constituents and unmineralised hyaline cartilage in intact tissue using BSE SEM.

\section{Acknowledgements}

The idea of block surface staining with triiodide arose following discussions with R.S. Stephenson and J.C. Jarvis, with whom I am working further on the development of the method for soft tissues.

I thank the reviewers for insightful comments and questions, Maureen Arora for technical assistance and The Veterinary Advisory Committee of the Horserace Betting Levy Board for earlier support which enabled us to generate the equine specimens.

\section{References}

Bab I, Ashton BA, Owen ME, Boyde A (1984) Incident light microscopy of surfaces of plastic embedded hard tissues. J Microsc 134: 49-53.

Bonfield W, Grynpas MD, Tully AE, Bowman J, Abram J (1981) Hydroxyapatite reinforced polyethylene - a mechanically compatible implant material for bone replacement. Biomaterials 2: 185-186.

Boyde A (2012) Scanning electron microscopy of bone. Methods Mol Biol 816: 365-400.

Boyde A, Firth EC (2005) Musculoskeletal responses of 2-year-old thoroughbred horses to early training. 8 . Quantitative back-scattered electron scanning electron microscopy and confocal fluorescence microscopy of the epiphysis of the third metacarpal bone. N Z Vet J 53: 123132.

Boyde A, Koole LH (2001) Embed in an iodinated polymer: a new paradigm for histology via backscattered electron imaging. J Anat 199: 217 abstract 1.

Boyde A, Reid SA (1983) Tetracycline cathodoluminescence in bone, dentine and enamel. Histochemistry 77: 525-533.

Boyde A, Wolfe LA (2000) Spatial and temporal patterns of bone growth around endosseous implants: A critical study of microscopic methods. Chapter 29 in "Bone Engineering” (Davies JE, Ed), em squared Inc., Toronto, Canada, pp 321-331.

Boyde A, Reid SA, Howell PGT (1983) Stereology of bone using both backscattered electron and 
cathodoluminescence imaging for the SEM. Beitr elektronenmikroskop Direktabb Oberfl (Muenster) 16: 419-430.

Boyde A, Howell PGT, Bromage T, Elliott JC, Riggs CM, Bell LS, Kneissel M, Reid SA, Jayasinghe JAP, Jones SJ (1992a) Applications of mineral quantitation of bone by histogram analysis of backscattered electron images. In: Chemistry and Biology of Mineralised Tissues, (Slavkin H, Price P, Eds) Excerpta Medica Int Congr Ser ICS 1002 Elsevier, Amsterdam, pp 47-60.

Boyde A, Wolfe LA, Jones SJ, Vesely P, Maly M (1992b) Microscopy of bone cells, bone tissue, and bone healing around implants. Implant Dent 1: 117-125.

Boyde A, Haroon Y, Riggs CM (1999a) Three dimensional structure of the distal condyles of the third metacarpal bone. Equine Vet J 31: 122-129.

Boyde A, Travers R, Glorieux FH, Jones SJ (1999b) The mineralisation density of iliac crest bone from children with osteogenesis imperfecta. Calcif Tiss Int 64: 185-190.

Boyde A, Howell PGT, Koole LH (2001) A new method for histology using backscattered electron imaging of tissue embedded in an iodinated polymer. Scanning 23: 75.

Boyde A, Lovicar L, Zamecnik J (2005) Combining confocal and BSE SEM imaging for bone block surfaces. Eur Cell Mater 26: 33-38.

Cox PG, Jeffery N (2011) Reviewing the morphology of the jaw-closing musculature in squirrels, rats, and guinea pigs with contrast-enhanced microCT. Anat Rec 294: 915 928.

Degenhardt K, Wright AC, Horng D, Padmanabhan A, Epstein JA (2010) Rapid 3D phenotyping of cardiovascular development in mouse embryos by micro-CT with iodine staining. Circ Cardiovasc Imaging 3: 314-322.

Ferguson VL, Bushby AJ, Boyde A (2003) Nanomechanical properties and mineral concentration in articular calcified cartilage and subchondral bone. J Anat 203: 191-202.

Hutchinson JR, Delmer C, Miller CE, Hildebrandt T, Pitsillides AA, Boyde A (2011) From flat foot to fat foot: structure, ontogeny, function, and evolution of elephant "sixth toes". Science 334: 1699-1703.

Jeffery NS, Stephenson RS, Gallagher JA, Jarvis JC, Cox PG (2011) Micro-computed tomography with iodine staining resolves the arrangement of muscle fibres. J Biomech 44: 189-192.

Metscher BD (2009a) MicroCT for comparative morphology: simple staining methods allow high-contrast 3D imaging of diverse non-mineralized animal tissues. BMC Physiol 9: 11-25.

Metscher BD (2009b) MicroCT for developmental biology: a versatile tool for high-contrast $3 \mathrm{D}$ imaging at histological resolutions. Dev Dyn 238: 632-640.

Newcomb GM, Boyde A (1980) SEM histochemistry - the use of backscattered electrons to identify epidermal Langerhans cells in the scanning electron microscope. Histochem J 12: 695-700.

Reid SA, Boyde A (1987) Changes in the mineral density distribution in human bone with age: Image Analysis using backscattered electrons in SEM. J Bone Min Res 2: 13-22.
Ritman EL (2011) Current status of developments and applications of micro-CT. Ann Rev Biomed Eng 13: 531552.

Stephenson RS, Boyett MR, Hart G, Nikolaidou T, Cai X, Corno AF, Alphonso N, Jeffery N, Jarvis JC (2012) Contrast enhanced micro-computed tomography resolves the 3-dimensional morphology of the cardiac conduction system in mammalian hearts. PLoS One 7: e35299.

Wong K (2000) Studies of the quality of the intraosseous dental implant bed and of thermal effects in implant pathology. PhD Thesis, University of London.

\section{Discussion with Reviewers}

Reviewer II: Do you need poor vacuum conditions?

Author: Not necessary, but very convenient because one can stain one sample, or a part of the surface of one sample at a time and not have to re-coat the sample with carbon and then wait longer for satisfactory vacuum conditions.

Reviewer II: Would the use of lower working distance values (with a suitable BSE detector) make suitable imaging possible as well?

Author: Considering an annular detector located close to the final lens in the sample chamber, the solid angle of BSE collected will increase as one reduces the working distance (a good thing), but then so will the field of view over which the beam is small enough for the image to be 'in focus' (a bad thing). I have found working distances in the range 8 to $12 \mathrm{~mm}$ to be a good compromise with the Zeiss EVO MA10 instrument.

Reviewer II: You have demonstrated the use of this technique to provide samples for BSE imaging at beam voltage of $20 \mathrm{kV}$. Have you attempted to obtain images using lower $\mathrm{kV}$ values, as this could potentially increase the resolution obtainable (due to reduced beam-spreading in the sample)?

Author: As you indicate, the volume within the electron beam is dissipated reduces with $\mathrm{kV}$, thus potentially increasing resolution (a good thing). However, concentrating the energy in a smaller volume also means that beam damage is increased, whilst the useful BSE signal is reduced (both bad things). I have tried lower $\mathrm{kV}$, with the expected consequences. I found $20 \mathrm{kV}$ to be a good compromise.

My experience in working with plastic embedded samples is that the main beam damage problem lies where the embedding medium is concentrated and there is little tissue solid. This happens, for example, outside the tissue proper, in sparse connective tissue and in adipocytes because the lipid has been removed in the embedding process.

Reviewer II: Clearly, the discussion revolves around handling PMMA-embedded material. There is a suggestion, both in the Abstract and Introduction, of the applicability of this method to material embedded in 'other plastic' blocks - but no examples are given or discussion included 
of the possible differences that might be found with other embedding materials, such as epoxy resins.

Author: True, all the examples I gave in the paper are pure PMMA embedded. In response to this query, I have checked that the method works with other resins commonly used with unstained (meaning no heavy element staining) massive tissue samples, so that I can now affirm that it works with glycol methacrylate, LR White, Technovit, butyl-methyl methacrylate mixtures and PMMA with added softener as commonly used for light microscopic histology. I have been unable to locate any unstained epoxy resin embedded tissue, but since many 'electron stains' are used with ultra-thin sections in TEM, I expect that the method would work, but have not proved it. However, my experience is that it is much more difficult to achieve a nice flat polish any of the aforementioned resins, including epoxies, than with PMMA.

Reviewer III: What kind of "stains" other than Lugol's iodine would be conceivably used in a similar way?
Which developments of the approach does the author see as possibly coming from using or manipulating existing histochemical methods?

Author: I tried conventional stains used for TEM sections, including uranyl acetate and lead citrate, on PMMA block faces. These work, but tend to lead to excess stain in polishing blemishes, and may also 'stain' mineralised areas so that the signal is even brighter, whereas what we need is to visualise low density features.

Any histochemical procedure which leads to the deposition of a heavy element containing end product can be used for BSE imaging at a resolution slightly better than that of light microscopy. However, most of these will be envisaged to work with unembedded tissue with the tissue being embedded afterwards, whilst we want to exploit existing masses of PMMA embedded material. The most likely exception will be the case of glycol methacrylate embedded blocks. However, as already noted, this material is very difficult to polish, and existing as-microtomed surfaces are very rough by the standards required for SEM. 